\title{
Application of Lasers in Orthodontics
}

SADJ July 2017, Vol 72 no 6 p276 - p277

G Melman', V Karic ${ }^{2}$, R Mulder ${ }^{3}$

Laser is the acronym for "Light Amplification by Stimulated Emission of Radiation," a usage that dates back to approximately 50 years ago. In 1960, the first functioning laser was built by the American physicist Maiman at the Hughes Research Laboratories by using a synthetic ruby crystal made of aluminum oxide and chromium oxide. In general, lasers are composed of the three principal parts: an energy source, an active medium and a set of two or more mirrors that form a resonator. Properties such as wavelength are determined primarily by the active medium, which can be a gas, crystal or a solid-state conductor.

In orthodontic practice, lasers have many common applications, including acceleration of tooth movement, bone remodeling, enamel etching prior to bonding, debonding of ceramic brackets, pain reduction after application of orthodontic forces and prevention of enamel demineralization. Soft-tissue applications such as frenectomies, gingival contouring and crown lengthening can also be achieved using the dental lasers. ${ }^{1}$

The proper use of a laser in an orthodontic office can accelerate treatment, reduce the number and length of appointments needed, and provide superior results. Two types of lasers may be used in orthodontic practice: soft tissue surgical lasers, which are used to incise/excise tissue; and photobiomodulating (PBM) lasers, which decrease posttreatment discomfort, favourably affect bone growth rate, and accelerate tooth movement.

One primary consideration important to orthodontists would be the ability to effectively treat soft tissue while avoiding alteration of hard tissues. Additional consider-

1. Geoffrey Melman: BDS(Wits), MSC(Dent)(Wits). Specialist in Paediatric Dentistry(U.K.), Department of Paediatric Dentistry, University of the Witwatersrand, School of Oral Health Sciences, Johannesburg.

2. Vesna Karic: $B D S(B g d, E U), M S c($ Dent)(Wits). Senior Lecturer Division of Operative Dentistry, University of the Witwatersrand, School of Oral Health Sciences, Johannesburg.

3. Riaan Mulder: $B C h D(U W C), \quad M S c(D e n t)(U W C)$. Lecturer Department of Orthodontics and Paediatric Dentistry. University of the Western Cape, Tygerberg, Cape Town.

\section{Corresponding author}

Vesna Karic:

Senior lecturer, Division of Operative Dentistry, University of the

Witwatersrand, School of Oral Health Sciences, Johannesburg, South

Africa, 7 York Road, Parktown, 2193. E-mail: xxxxxx@wits.ac.za

\section{ACRONYMS}

Laser: Light Amplification by Stimulated Emission of Radiation PBM: photobiomodulating

ations might be portability and cost. Manufacturers claim all wavelengths are capable of performing soft tissue procedures, but some wavelengths, such as those emitted by the erbium family of lasers (Er:YAG,Er,Cr:YSGG), are also promoted as suitable for hard tissue procedures. These lasers usually require water cooling, so they tend to be bulky and relatively expensive in comparison with lasers producing other wavelengths. Carbon dioxide $\left(\mathrm{CO}_{2}\right)$ laser wavelengths are well absorbed by water, and because oral mucosa is more than $90 \%$ water, $\mathrm{CO}_{2}$ lasers are very effective soft tissue lasers. In addition, $\mathrm{CO}_{2}$ lasers often have a "superpulse" feature, providing relatively high energy in short spurts, offering a very efficient means to ablate soft tissue. On the other hand, $\mathrm{CO}_{2}$ lasers often are larger and more expensive than diode lasers which are currently available in four distinct wavelengths (810 to $830,940,980$, and $1064 \mathrm{~nm})$. Neodymium-doped yttrium-aluminum-garnet (Nd:YAG) lasers (1064nm) emit energies that are well absorbed by haemoglobin and melanin, so these lasers work well for ablation of pigmented and vascular tissues such as oral mucosa. Diode lasers have a much shallower depth of penetration than Nd:YAG lasers and may be less likely to cause pulpal damage, making them an excellent choice in orthodontics. In addition, diode lasers tend to be the least expensive and most portable of lasers of all wavelengths-another considerable advantage in orthodontics. ${ }^{2}$

Many procedures can be done with a soft tissue laser in orthodontic practice, but most fall into two categories: access gingivectomies and aesthetic procedures.

Access gingivectomies involve exposing more tooth tissue for earlier or more ideal bracket or band placement. Aesthetic procedures involve removing redundant gingival tissues to optimize gingival aesthetics and to improve the appearance of completed orthodontic cases. In the performance of these procedures safely and efficiently, with the least discomfort for the patient, it is important to know what not to do. Three primary considerations should be kept in mind in performing soft tissue laser procedures: biologic width or zone, pocket depth, and keratinized 
tissue. For gingival aesthetic procedures, several additional diagnostic considerations are of importance as well: Access to partially erupted teeth, access to unerupted teeth, access for ideal bracket placement and enhancing access in gingival hyperplasia resulting from poor oral hygiene. Additionally lasers can be applied for other orthodontic procedures such as: labial frenum removal, lingual frenum removal and aphthous ulcer pain relief. (Aphthous ulcers can be extremely painful, and laser therapy can relieve pain and speed healing). The vast majority of laser procedures in an orthodontic office may be performed using topical anaesthesia. ${ }^{2}$

\section{PHOTOBIOMODULATING LASERS IN ORTHODONTICS}

PBM lasers by definition are nonsurgical-that is, they are incapable of cutting tissues. As their name implies, they use light (photo) energy to biologically modulate cellular functions.

\section{CONCLUSIONS}

Lasers are considered as "light at the end of the tunnel" in dentistry. It has been proven that when used effectively and ethically, lasers can be an exceptional modality of treatment for many clinical conditions. There are many advantages of lasers which include improved oral hygiene and aesthetic finishing. An adjunctive procedure such as lasers can dramatically enhance the entire procedure in the practice of an orthodontist who is committed to provide the best possible service. ${ }^{3}$

\section{References}

1. Nalcaci R, Cokakoglu S. Lasers in orthodontics Eur J Dent. 2013 Sep; 7(Suppl 1): S119-S125.

2. Convissar A. R, Principles and Practice of Laser Dentistry, Elsevier 2016

3. Khajuria AK, Prasantha GS, Mathew S, Khan Y et al. Lasers in Orthodontics, Journal of Dental \& Oro-facial Research 2016,12 (02)

\section{(c)}

(6) (6) (y) (y) JONUS NNEW ZEALANDANDHAVEA LIFESTYLETO ENM

\section{NEW ZEALAND PASSENGER ARRIVAL CARD}

Rated in the top ten safest countries in the world - New Zealand is the place where you can enjoy beaches, parks, award-winning wine, world-class restaurants and a range of cultures right on your doorstep - literally.

New Zealand has that perfect combination of big city and country town all rolled into one. With a great community feel, excellent schooling and a thriving economy - New Zealand really is a place that you can call home.

With 91 practices nationwide there are opportunities everywhere to enjoy the lifestyle you want, plus enjoy:

Excellent salary, standard of living and work/life balance

(d) Lucrative earning potential in smaller towns

(d) Career advancement and development within the Lumino Group

1 We need talented

to start

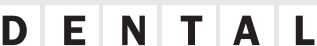

\begin{tabular}{lllll|l|l|l|l|l|l} 
C & $\mathbf{L}$ & I & $\mathbf{N}$ & I & C & I & $\mathbf{A}$ & $\mathbf{N}$ & $\mathbf{S}$
\end{tabular}

A S A $\mathrm{A}$ P

2 For further information:

\begin{tabular}{lllll|l|l|l|l|l|l|l|l|l|l|l|l|l|l|l|l} 
C & A & $\mathbf{R}$ & $\mathbf{E}$ & $\mathbf{E}$ & $\mathbf{R}$ & $\mathbf{S}$ &. & $\mathbf{L}$ & $\mathbf{U}$ & $\mathbf{M}$ & $\mathbf{I}$ & $\mathbf{N}$ & $\mathbf{0}$ &. & $\mathbf{C}$ & $\mathbf{0}$ &. & $\mathbf{N}$ & $\mathbf{Z}$
\end{tabular} 